\title{
Accurate Grain and Phase Boundary Location by Dictionary-based Indexing of Geological EBSD Data
}

\author{
Saransh Singh ${ }^{1}$, Katharina Marquardt ${ }^{2}$, and Marc De Graef ${ }^{1}$ \\ 1. Dept. of Materials Science and Engineering, Carnegie Mellon Univ., Pittsburgh, PA, USA \\ 2. Bayerishes Geoinstitut, University Bayreuth, Bayreuth, Germany
}

Dictionary-based indexing (DI) is a recent approach [1] to the indexing of electron diffraction pat-terns, including electron backscatter diffraction (EBSD), in which a library of precomputed patterns is compared to a series of experimentally acquired patterns. The library is generated using an accurate physics-based forward model that incorporates the geometry of the sample-detector configuration as well as the dynamical and stochastic nature of the generation of backscattered electrons (BSEs) in the sample. Combined with a uniform sampling of orientation space, $S O(3)$, DI uses a similarity metric (normalized dot product between pattern vectors) to select from the dictionary the pattern that best matches the experimental pattern; the orientation corresponding to the best match is assigned as the orientation of the experimental pattern. Since a similarity measure is obtained for each dictionary en-try, one can rank the top $N$ matches and store them for further analysis; below we show that an analysis of the common matches in this set provides an accurate location of grain and phase boundaries, which are often incorrectly indexed using Hough transform based methods due to pattern overlaps.

Consider a set of experimental EBSD patterns reorganized as normalized 1D vectors, $\left\{\hat{\mathbf{e}}_{i}\right\},\left(i \in\left[1, N_{e}\right]\right)$, and a dictionary consisting of normalized vectors $\left\{\hat{\mathbf{d}}_{j}\right\}\left(j \in\left[1, N_{d}\right]\right)$. For each experimental pattern with sampling coordinates $(\mu, \nu)$, a ranked set of dot products $\mathcal{D}_{\mu, \nu}=\left\{\hat{\mathbf{e}}_{\mu, \nu} \cdot \hat{\mathbf{d}}_{j}\right\}\left(j \in\left[1, N_{d}\right]\right)$ is computed (ranked from high to low). The top $N$ (typically $N=20$ ) dot product values and the corresponding set of orientation labels $\mathcal{S}_{\mu, \nu}=\{j\}$ are stored for further analysis. We define the Ori-entation Similarity value, $\Omega_{\mu, \nu}$, of an experimental pattern $(\mu, \nu)$ as the average of the cardinalities of the intersections $(\cap)$ of the set $\mathcal{S}_{\mu, \nu}$ with the sets of the four nearest neighbors of $(\mu, \nu)$ :

$$
\Omega_{\mu, \nu}=\frac{1}{4}\left[\#\left(\mathcal{S}_{\mu, \nu} \cap \mathcal{S}_{\mu+1, \nu}\right)+\#\left(\mathcal{S}_{\mu, \nu} \cap \mathcal{S}_{\mu-1, \nu}\right)+\#\left(\mathcal{S}_{\mu, \nu} \cap \mathcal{S}_{\mu, \nu+1}\right)+\#\left(\mathcal{S}_{\mu, \nu} \cap \mathcal{S}_{\mu, \nu-1}\right)\right]
$$

where $\#(A)$ represents the cardinality of set $A$. Fig. 1(a) and (b) show the orientation similarity maps (OSMs) for a three-phase nominally anhydrous residual eclogite, consisting of 37\% (cubic) garnet (grt), $39 \%$ (monoclinic) clinopyroxene (cpx), and an amorphous melt phase (24\%); the phase map in (c) identifies the three phases (grt=red, $\mathrm{cpx}=$ blue, melt=yellow). The OSM has a high value for points inside a grain, and decreases rapidly at grain boundaries.

Individual grain or phase boundaries can now be located accurately by considering how the orienta-tion similarity value changes along a line traversing the boundary. Fig. 2(a) shows a portion of the cpx OSM of Fig. 1(a) along with a line trace across the grain boundary between grains $A$ and $B$. If we label the points along the line by the index $k$, with $k=0$ corresponding to the starting point of the line in grain $A$, then we define the Linear Orientation Similarity (LOM), $\lambda_{k}(A)$, as the cardinality of the intersection of the sets $\mathcal{S}_{A}$ and $\mathcal{S}_{k}$. For $N=20$, the number of common orientation matches oscillates near this value for points that lie inside grain $A$, and suddenly drops to zero when the grain boundary is crossed, since the set $\mathcal{S}_{A}$ has no matches in common with grain $B$; the profile $\lambda_{k}(A)$ is shown in Fig. 2(b). Similarly, we determine the $\operatorname{LOM} \lambda_{k}(B)$ by traversing the line starting at the end point in grain $B$; once again, the LOM value drops to zero upon crossing the boundary. 
The point where the two LOM curves intersect each other, i.e., $\lambda \mathrm{k}(\mathrm{A})=\lambda \mathrm{k}(\mathrm{B})$, can be considered to be an estimate of the location of the grain boundary. It is clear from the figure that this location can be determined towithin a fraction of the pixel spacing, which represents a significant improvement over the ability of the Hough transform-based indexing techniques to locate grain and phase boundaries [2].

References:

[1] Y.H. Chen et al, Microsc. MicroAnal. 21 (2015), p. 739.

[2] Research supported by AFOSR-MURI \# FA9550-12-1-0458. The authors acknowledge use of the Materials Characterization Facility at Carnegie Mellon University supported by grant MCF-677785.

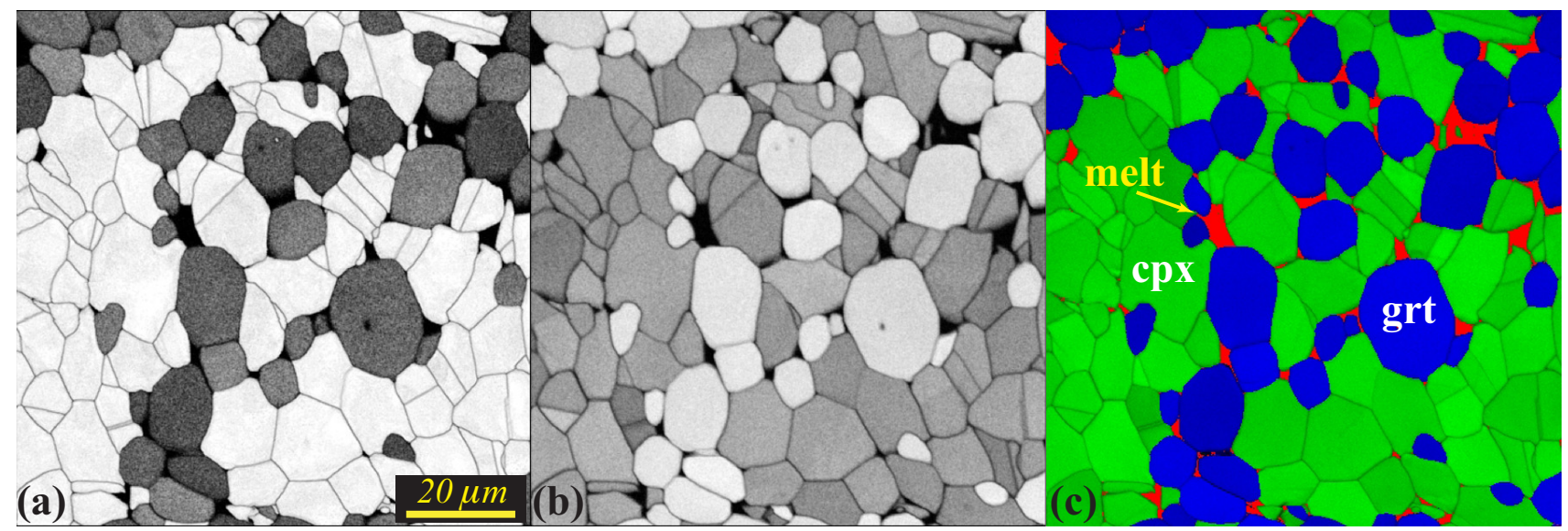

Figure 1. Orientation similarity maps (OSMs) for cpx (a) and grt (b), along with a phase map in (c) [blue $=$ grt, green $=c p x$, red=melt $]$. Each OSM highlights grains that belong to the corresponding phase
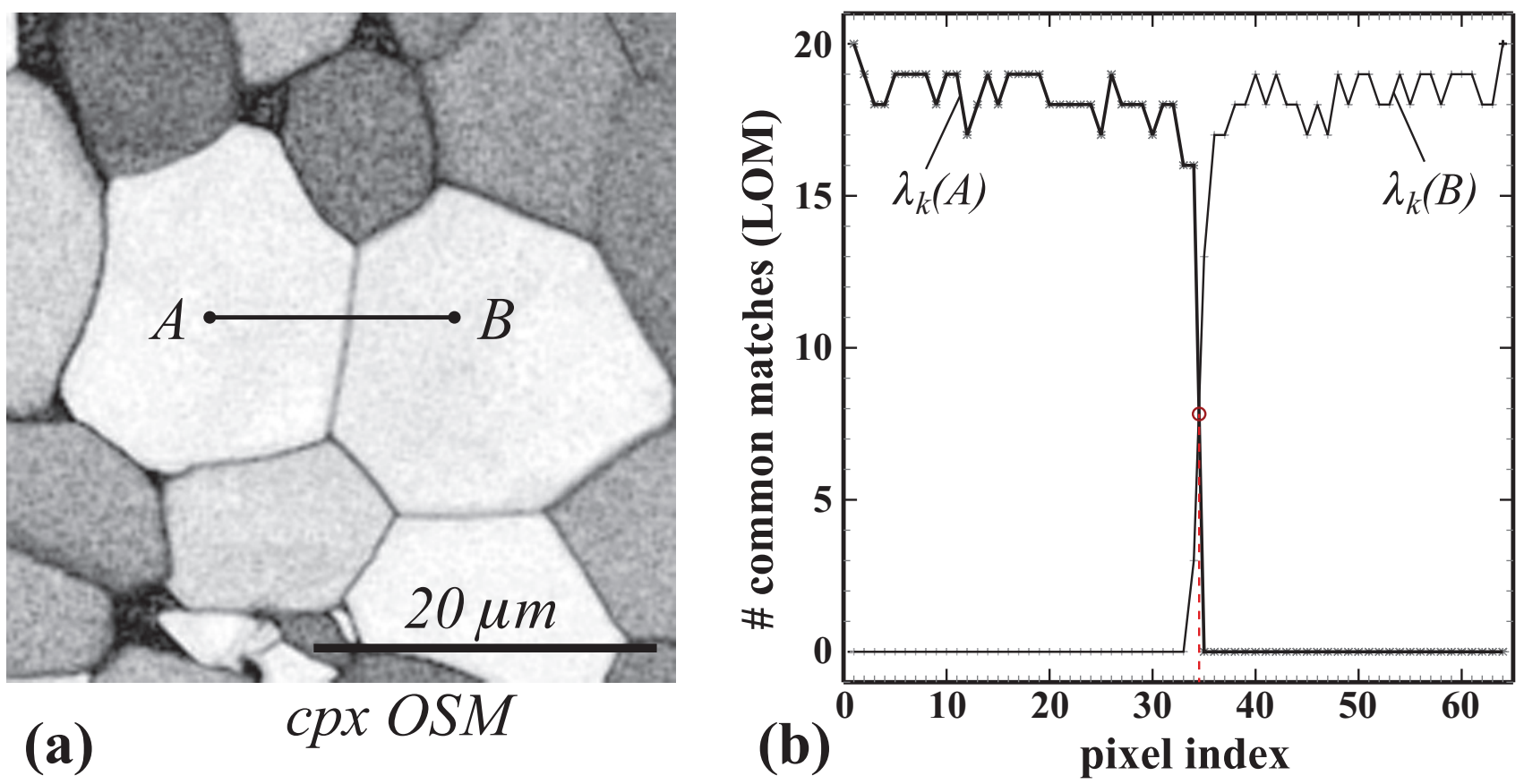

Figure 2. (a) portion of the OSM from Fig. 1(a) with a trace line across the grain boundary between grains $A$ and $B$; (b) linear orientation similarities $\lambda_{k}(A)$ and $\lambda_{k}(B)$. The intersection of the two profiles indicates the location of the grain boundary. 